\title{
FLOW AROUND INDIVIDUAL TAYLOR BUBBLES RISING IN STAGNANT CMC SOLUTIONS: PIV MEASUREMENTS
}

\author{
R. G. Sousa*, A. M. F. R. Pinto*, M. L. Riethmuller**, J. B. L. M. Campos** \\ ** Departamento de Engenharia Química, Centro de Estudos de Fenómenos de Transporte, \\ Faculdade de Engenharia da Universidade do Porto, \\ Rua Dr. Roberto Frias 4200-465 Porto, Portugal, E-mail: jmc@fe.up.pt \\ *** von Karman Institute for Fluid Dynamics, \\ 72 Chaussée de Waterloo, 1640 Rhode-Saint-Genèse, Belgium, E-mail: riethmuller@vki.ac.be
}

\begin{abstract}
The flow around single Taylor bubbles rising in non-Newtonian solutions of Carboxymethylcellulose (CMC) polymer was studied using a simultaneous technique employing particle image velocimetry (PIV) and shadowgraphy. This technique solved previous problems on finding the correct position of the bubble interface. Solutions of different polymer weight percentage, varying from 0.1 to $1.0 \mathrm{wt} \%$ were used to cover a wide range of flow regimes. The rheological fluid properties and pipe dimension yielded Reynolds numbers between 4 and 254 and Deborah numbers between 0.012 and 0.402 . The shape of the bubbles in the different fluids was compared. The flow around the nose of the bubbles was found to be similar in all the studied conditions. Velocity profiles in the liquid film around the bubble were measured. Different wake structures were found in the different solutions studied. With increasing viscosity, the wake flow varied from turbulent to laminar, being possible to observe a negative wake for the higher polymer concentration solutions. A comparison between the different wake structures was made.
\end{abstract}

\section{INTRODUCTION}

Slug flow is a two-phase flow regime found when a gas and a liquid flow simultaneously in a pipe over certain flow rates. It is characterized by elongated gas bubbles (Taylor bubbles or slugs) almost filling the pipe cross section and liquid flowing around and between the bubbles. This flow pattern is found in several industrial processes as is the case of geothermal, oil and gas wells, fermentation, polymer devolatilization and airlift reactors among others. In some chemical processes, slug flow is induced to increase the reaction rate due to the mixing created mainly in the wake of the Taylor bubbles.

The velocity of the bubble depends among other things, in the velocity of the liquid ahead of it [1]. When two bubbles flow together in a pipe, the wake of the leading one can influence the velocity of the following, if the distance between them is smaller than the minimum stable length [2]. In this case, two bubbles can merge together (coalescence) forming a larger bubble and reducing the number of mixing zones and therefore the process efficiency. Coalescence is therefore a problem to avoid and the minimum stable distance between two consecutive bubbles is essentially dependent on the flow in the wake of the preceding bubble.

With newtonian fluids, among other studies, Campos and Guedes de Carvalho [3] studied wake structure for different liquid flow regimes. Pinto and Campos [4] studied the interaction between two consecutive bubbles and established minimum stable lengths for different operation conditions. PIV measurements were made to characterize the flow field around individual Taylor bubbles in newtonian fluids [5, 6, 7, 8].

The study of Taylor bubbles rising in non-Newtonian liquids is still scarce, although they are found frequently in industry.
Due to the complex liquid rheology, the gas-liquid flow patterns have completely different characteristics, and by consequence different bubble interaction behavior. Therefore, there is a need for extending the slug flow research towards nonNewtonian liquids. Some studies have been made with small bubbles in non-Newtonian liquids. Bubble velocities and shapes in non-Newtonian liquids have been studied by different authors $[9,10,11]$. Hassager [12] described, for the first time, a negative wake behind unconfined small bubbles rising in non-Newtonian liquids. Bubbles coalescence studies in non-Newtonian liquids can be found in [13, 14, 15]. Funfschilling and $\mathrm{Li}[16]$ used PIV and birefringence visualization to study the flow of nonNewtonian fluids around small bubbles.

Concerning the flow of Taylor bubbles in non-Newtonian fluids, the effects of power law rheology and pipe inclination on slug bubble rise velocity were studied by Carew et al. [17]. Otten and Fayed [18] studied the pressure drop and friction drag reduction in two-phase non-Newtonian slug flow. Rosehart $e t$ al. [19] measured the void fraction, slug velocity and frequency for co current slug flow of air bubbles in highly viscous nonNewtonian fluids. Terasaka and Tsuge [20] performed gas holdup measurements for slug bubbles rising in viscous liquids having a yield stress.

In the present work, the flow around single Taylor bubbles rising in non-Newtonian Carboxymethylcellulose solutions is studied, using PIV and shadowgraphy simultaneously. The results obtained contribute for a better understanding of the nonNewtonian behavior and the bubble coalescence mechanism. 


\section{EXPERIMENTS}

\subsection{Experimental Techniques}

The flow field around the Taylor bubbles rising in stagnant liquid was obtained applying PIV simultaneously with shadowgraphy. This technique was first presented by Lindken and Merzkirch [21] for bubbly flow and Nogueira et al. [22] adapted it for slug flow. The technique is fully described in $[22,23]$ and consists in placing a board of light emitting diodes (LEDs) behind the test section pulsing simultaneously with the laser, so that the CCD camera, acquires an image containing both the PIV particles and the shadow of the bubble. Two lenses of $35 \mathrm{~mm}$ and $50 \mathrm{~mm}$ of focal length was used to obtain the flow field in the nose/wake region and to obtain a close view of the liquid film around the bubble. The LEDs board is composed by 350 LEDs. Fluorescent particles (an orange vinyl pigment, $10 \mu \mathrm{m}$ of mean size) were used as seeding and emitted light at $590 \mathrm{~nm}$. A Nd: YAG laser was used to create a vertical laser sheet of about $1 \mathrm{~mm}$ thickness containing the axis of the column. The laser, the camera and the LED's board were all triggered by the same signal generator which makes possible to obtain the PIV images and the bubble shadow in the same frame.

Two laser diodes were placed at a fixed distance in the column pointing through the center into two photocells in the opposite side of the column. The signal yielded by the photocell is proportional to the light received, so when the Taylor bubble is passing between a laser diode and a photocell, the laser beam is deflected and the photocell signal drops abruptly. The Taylor bubble velocity was determined dividing the distance between the photocells by the time delay between their signals.

\subsection{Facility}

The present study was made in the experimental setup schematized in Figure 1 and fully described in [22, 23]. The

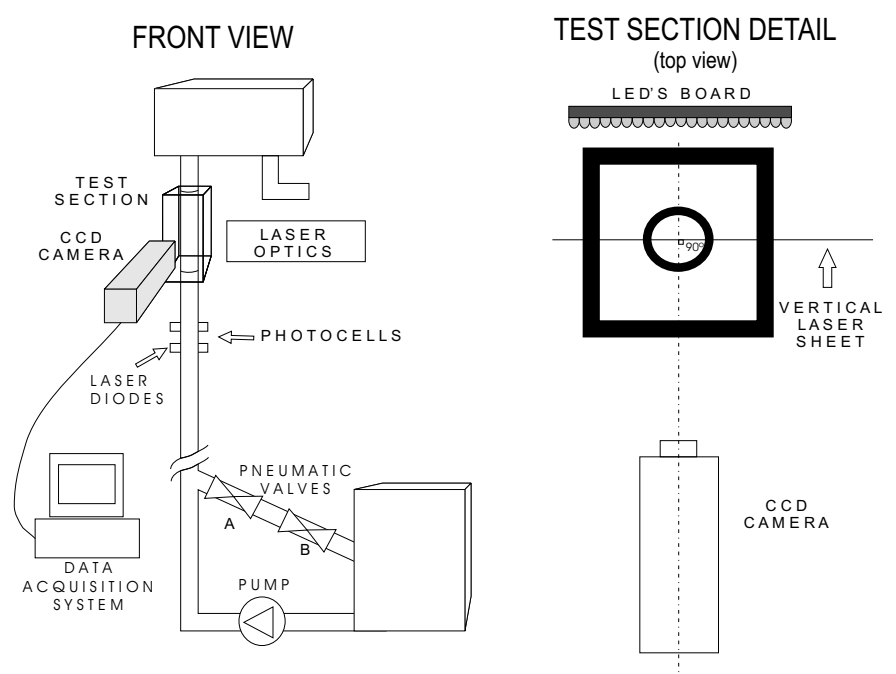

Figure 1. General scheme of the experimental setup and detailed top view of the test section

facility is mainly composed by an acrylic column of $6 \mathrm{~m}$ height and $0.032 \mathrm{~m}$ of internal diameter, open on top to the atmosphere, two pneumatic valves, two storing tanks and a pump. The test section was located near the top of the column, to avoid entrance effects and to assure a stabilized flow. A box with plane faces surrounding the test section $(0.5 \mathrm{~m} \times 0.12 \mathrm{~m} \times 0.11 \mathrm{~m})$ was filled with the studied liquid in order to minimize the optical distortion. The individual Taylor bubbles were injected at the bottom of the column, by manipulating valves A and B. The PIV instrumentation was basically composed by a PCO (SensiCam) CCD camera and an acquisition and data processing system. A Nd: YAG laser with $400 \mathrm{mj}$ of pulse power was used to illuminate the measured plane. The laser wavelength was $532 \mathrm{~nm}$ and the pulse duration $2.4 \mathrm{~ns}$. A LED array emitting light at $650 \mathrm{~nm}$ was placed behind the test section with a diffuser paper to obtain the shadow of the Taylor bubble at the same time as the PIV image. A red filter, opaque below $550 \mathrm{~nm}$ was placed in front of the PCO CCD camera to block the intense green reflections of the laser and to allow the passage of the light emitted by the fluorescent particles and by the LEDs. Two thermocouples were placed below and above the test section to determine the working temperature and to check a possible temperature gradient along the column. The fluid rheology was determined in a AR 2000 DTA Instruments Rheometer.

\section{DATA PROCESSING}

The images acquired with the simultaneous PIV and shadowgraphy technique contain both the PIV particles images and the shadow of the bubble in the same frame. The PIV processing method is fully described by Nogueira et al. [22]. The flow field was obtained using the cross-correlation algorithm WIDIM (Window Displacement Iterative Multigrid), developed by Scarano and Riethmuller [24]. In this work, initial interrogation windows had 20 pixels $x 40$ pixels and after the first vector estimative, final windows 10 pixels x 20 pixels were used. An interrogation areas overlap of $50 \%$ was used. Spurious vector identification was used, correcting vectors with a signal to noise ratio (SNR) less than 1.5 (about $5-7 \%$ of the total vectors) with the neighbors average value. The time between PIV images was adjusted according to the liquid velocity and varied between $1000-2000 \mu$ s for the wake and nose regions and $80-400 \mu$ s for the liquid film around the bubble. The estimated uncertainty of the measured liquid velocity is $2 \%$.

With the shadow of the bubble it is possible to identify the position of the gas-liquid interface and so overcome the problems described by Nogueira et al. [6] due to the reflection and refraction of particles images in the interface. The image processing for determination of the shadow of the bubble is described in previous works $[22,23]$ and consists of several sequencing steps. A median filter is applied to the original image to eliminate the seeding particles. A background reference image is then subtracted to the filtered image to eliminate the column and the background and the result is the shadow of the bubble. The process is completed by defining a gray level threshold, which binaries the image, and by filling the interior of the Taylor bubble. With the bubble shadow, it is then possible to identify the gas-liquid interface and eliminate the erroneous vectors inside the Taylor bubble.

\section{RESULTS}

In this work the flow around Taylor bubbles rising in stagnant solutions of Carboxymethylcellulose (molecular mass of $300000 \mathrm{~kg} \mathrm{kmol}^{-1}$ ) with different weight percentages was studied. In Figure 2 are represented the viscosities of the differ- 
ent solutions in function of the shear rate, measured in a AR 2000 DTA Instruments Rheometer. Due to difficulties in direct

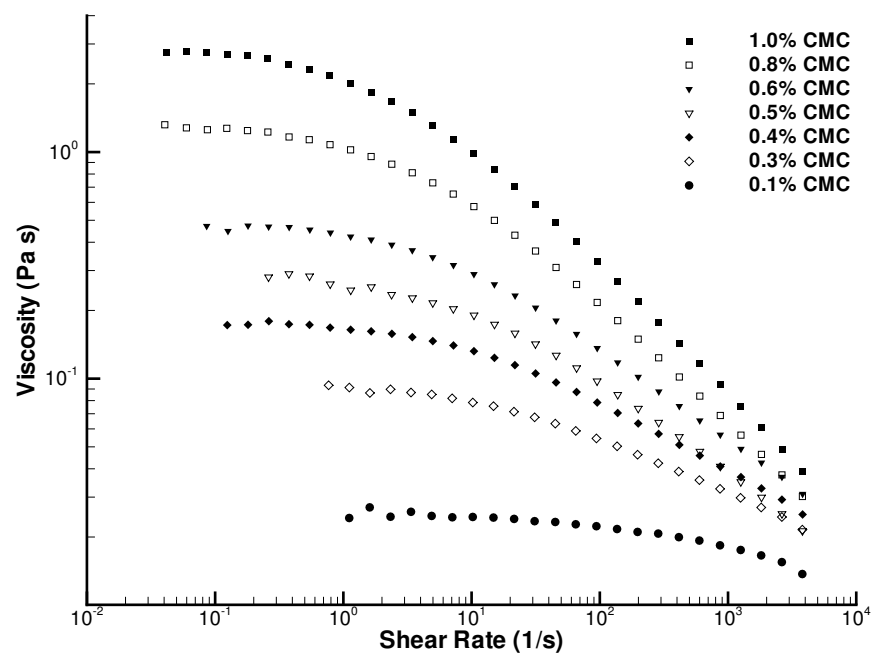

Figure 2. Representation of viscosity in function of the shear rate for the solutions studied

measurement, the relaxation times of the fluids were approximated to the fluid characteristic time, $\lambda$, of the Carreau simplified model, Eq. (1), after adjustment to the measured viscosities.

$$
\mu=\mu_{0}\left(1+(\lambda \cdot \dot{\gamma})^{2}\right)^{\frac{n-1}{2}}
$$

The experiments were made at ambient temperature. The experimental conditions are resumed in Table 1. The Reynolds

Table 1. Experimental temperature, bubble velocities and dimensionless $R e$ and $D e$ numbers

\begin{tabular}{c|cccc}
\hline $\mathrm{wt} \%$ & $\mathrm{~T}\left({ }^{\circ} \mathrm{C}\right)$ & $U_{b}(\mathrm{~m} / \mathrm{s})$ & $R e$ & $D e$ \\
\hline 0.1 & 20.3 & 0.199 & 254 & 0.012 \\
0.3 & 21.5 & 0.198 & 76 & 0.053 \\
0.4 & 19.0 & 0.195 & 44 & 0.092 \\
0.5 & 25.0 & 0.192 & 29 & 0.096 \\
0.6 & 19.0 & 0.187 & 18 & 0.192 \\
0.8 & 22.0 & 0.180 & 8 & 0.402 \\
1.0 & 22.0 & 0.160 & 4 & 0.374
\end{tabular}

number is defined as $R e=\rho U_{b} D / \mu_{b}$, where $\mu_{b}$ is the viscosity of the fluid obtained from the rheological data (figure 2), at a characteristic bubble shear rate $\left(\dot{\gamma}_{b}=U_{b} / D\right)$. The velocity field around the Taylor bubbles and its shape in the different conditions studied are described in the next sections.

\subsection{Taylor Bubble Shape}

The Taylor bubbles are elongated bubbles characterized by prolate spheroid leading edge, whose curvature is higher for higher viscosities. The bubble nose shapes, obtained from the bubble shadows are represented in Figure 3 for some of the conditions studied, in the format of dimensionless radius $(r / D)$ as a function of the dimensionless distance to the nose $(z / D)$, where $z$ is the distance from the nose, being positive downwards.

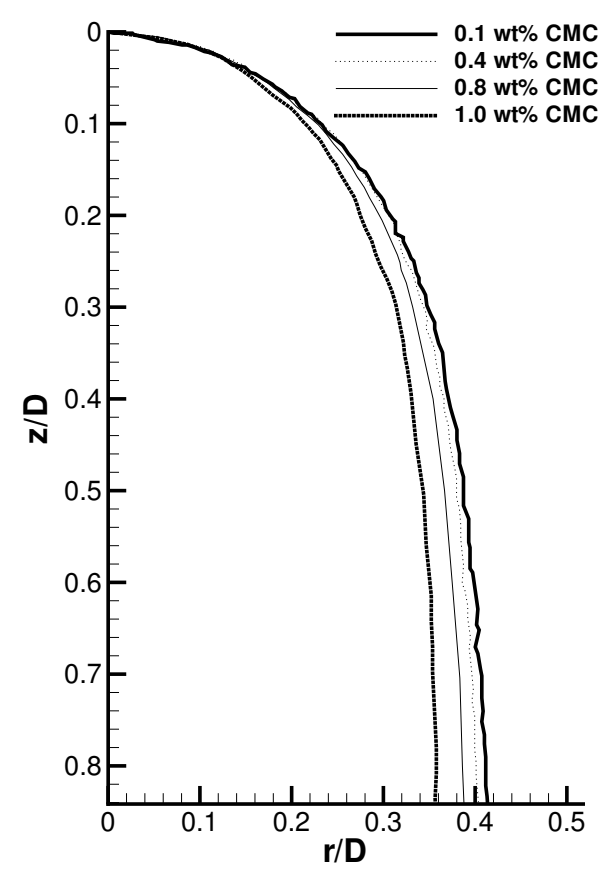

Figure 3. Representation of the bubble radius, $r / D$ in function of the distance to bubble nose, z/D.

The radius of the bubble increases with $\mathrm{z} / \mathrm{D}$, until it reaches a maximum value at a certain distance from the nose. The maximum bubble radius in each solution studied are presented in Table 2.

Table 2. Maximum bubble radius for the different solutions studied

\begin{tabular}{cccccc}
\hline $\mathrm{wt} \%$ & $(\mathrm{r} / \mathrm{D})_{\max }$ & & $\mathrm{wt} \%$ & $(\mathrm{r} / \mathrm{D})_{\max }$ \\
\cline { 1 - 2 } \cline { 5 - 6 } 0.1 & 0.462 & & 0.6 & 0.414 \\
0.3 & 0.446 & & 0.8 & 0.400 \\
0.4 & 0.435 & & 1.0 & 0.360 \\
0.5 & 0.426 & & &
\end{tabular}

The bottom of the bubble is where the main differences appear. Some PIV images of the bottom of the bubble are represented in Figure 4. For the higher Reynolds number (Figure 4 $\mathrm{a}, \mathrm{b})$, the bottom surface is unstable and oscillates as the bubble rises. As the viscosity increases, the trailing edge becomes stable and with a concave shape (Figure $4 \mathrm{c}, \mathrm{d}$ ). For the lower Reynolds numbers (Figure $4 \mathrm{e}, \mathrm{f}$ ), the trailing edge loses its concavity and gets a lacrimal shape. 


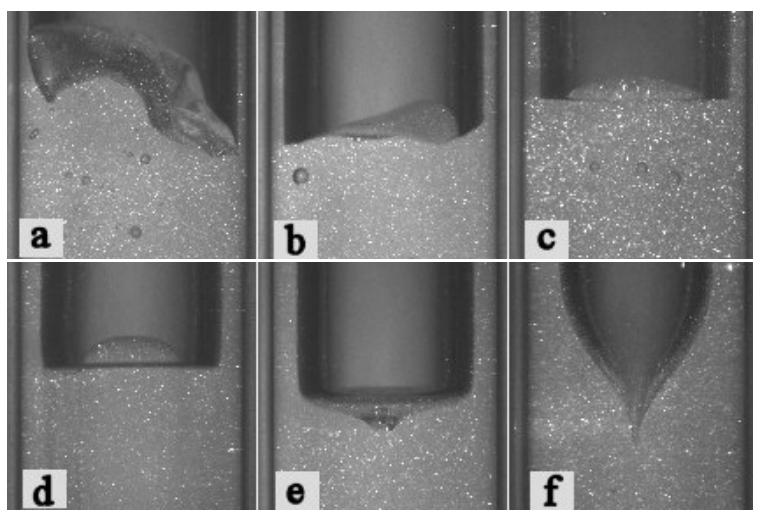

Figure 4. Bubble trailing edges images for a) $R e=254$, b) $R e=76$, c) $R e=44, \mathrm{~d}) \operatorname{Re}=18, \mathrm{e}) \operatorname{Re}=8$, f) $R e=4$.

\subsection{Flow Field in the Bubble Nose}

The flow field around the Taylor bubbles was obtained using Particle Image Velocimetry. The vector fields represented in the next sections refer to a vertical plane that passes in the center of the pipe.

The flow pattern around the nose of the bubbles is similar for every solution studied, varying only in the velocity magnitudes. Figure 5 shows the flow field around the nose of a Taylor bubble rising in a $0.5 \mathrm{wt} \% \mathrm{CMC}$ solution. The velocity vectors are relative to a fixed reference frame and the Taylor bubble is moving upwards. From Figure 5 it is possible to see that as the bubble

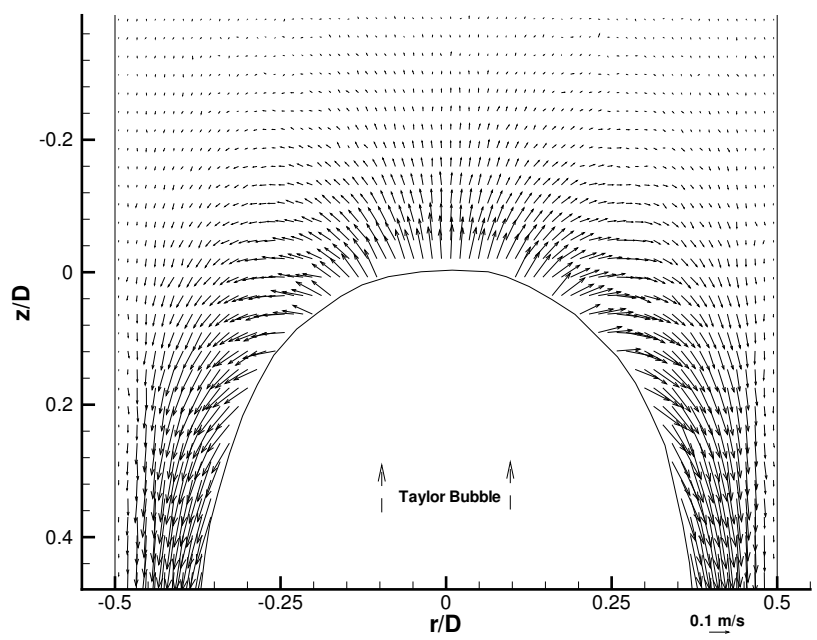

Figure 5. Flow around the nose of the Taylor bubble in $0.5 \mathrm{wt} \%$ solution

passes, the liquid in front of the bubble is pushed forward and away from the center and then starts falling around the bubble forming a thin liquid film.

In Figure 6 are represented two instantaneous vertical velocity profiles along $z=0$ for the two extreme cases studied. From Figure 6 , it is possible to see that the velocity profiles at $\mathrm{z}=0$ for the extreme cases are very similar, varying slightly the velocity magnitude due to the different bubble velocities and shapes. The velocity profiles correspondent to the other solutions studied are between these two, so they were not plotted for better graphic visualization.

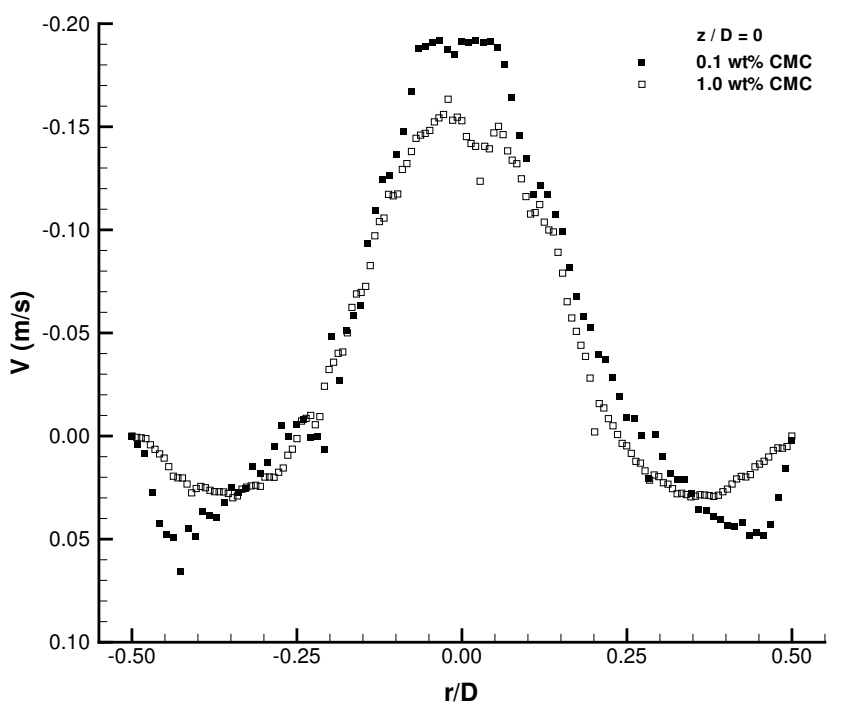

Figure 6. Vertical component of the liquid velocity along $z=0$ in 0.1 and 1.0 wt\% solutions

\subsection{Liquid Film Velocity Profile}

The liquid that flows around the bubble nose forms a liquid falling film between the gas-liquid interface and the pipe wall. The thickness of the liquid film decreases for higher values of $z$ according to the bubble radius, until it reaches a minimum thickness, where the liquid velocity profiles are fully developed. In Figure 7 are represented the average developed liquid velocity profiles for the conditions studied. From the plots in Figure 7

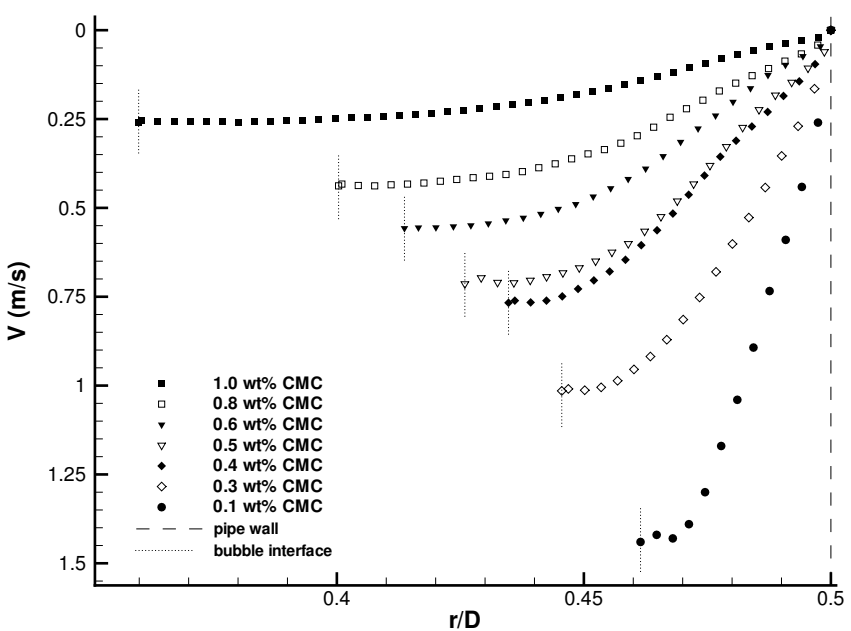

Figure 7. Average of the vertical component of the velocity in the liquid falling film in the different studied conditions

it is possible to see that the liquid film thickness is higher for higher $R e$ numbers. For the lowest viscosities, due to the thinner liquid film, the liquid velocity increases significantly, reaching up to 7 times the bubble velocity. 


\subsection{Taylor Bubble Wake}

The Taylor bubble wake is the region where the main differences appear in the flow pattern of the studied cases. For the higher values of $R e$, the liquid film velocity is higher and due to the low viscosity, the annular jet that falls behind the bubble causes a highly turbulent flow in the wake, responsible for the instability of the bubble trailing edge. The wake instability occurs for values of Reynolds higher than 44 and is characterized by oscillations of the bubble trailing edge which cause changes in time on the wake flow field. Figure 8 shows an instantaneous velocity field, relative to a fixed reference frame, in the wake of a Taylor bubble rising in a $0.1 \mathrm{wt} \%$ solution $(R e=254)$, where the variable $z *$ is the vertical distance to the center of the trailing edge of the bubble. From Figure 8 it is possible to identify some

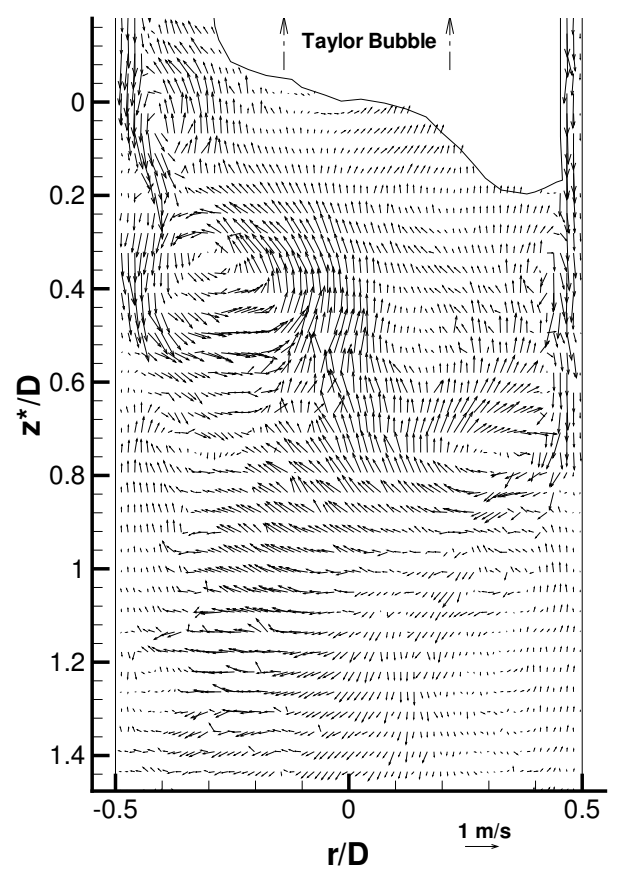

Figure 8. Instantaneous velocity field in the wake of a Taylor bubble rising in a $0.1 \mathrm{wt} \%$ solution

small vortices in the wake of the bubble. It should be noticed that the velocity field is obtained from a vertical plane in the center of the column and due to the high turbulent flow in this case, there is a lot of liquid movement in the plane perpendicular to the measurement plane. The maximum upward velocity in the wake can reach up to 4 times the bubble velocity, so liquid is transported upwards in the wake although it is not the same fluid that follows the bubble all the time, due to the high instability and vortex shedding.

As the viscosity increases, the liquid film velocity decreases and the wake instabilities due to the turbulence tend to disappear. For the $0.3 \mathrm{wt} \%$ solution $(R e=76)$, there is still some instability in the trailing edge of the bubble but much less than in the $0.1 \mathrm{wt} \%$ case. In Figure 9 is represented the flow field in the wake of a Taylor bubble rising in $0.3 \mathrm{wt} \%$ solution and although the trailing edge still oscillates, it is already possible to see a large asymmetric vortex.

In the $0.4 \mathrm{wt} \%$ solution $(R e=44)$, the Taylor bubble trailing edge and the wake flow are already stable, as can be seen in

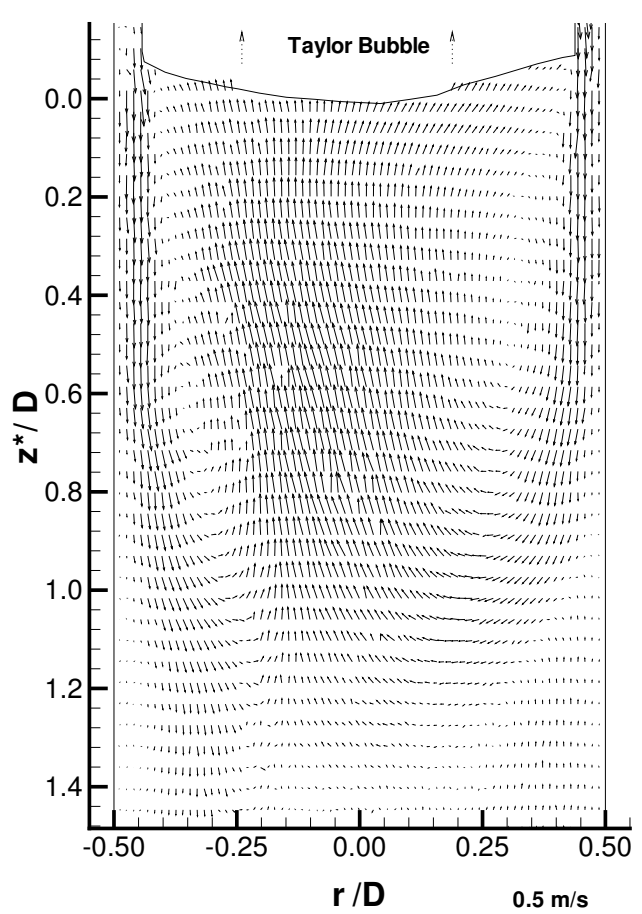

Figure 9. Instantaneous velocity field in the wake of a Taylor bubble rising in a $0.3 \mathrm{wt} \%$ solution

Figure 10. The wake flow pattern is now stable and symmetric,

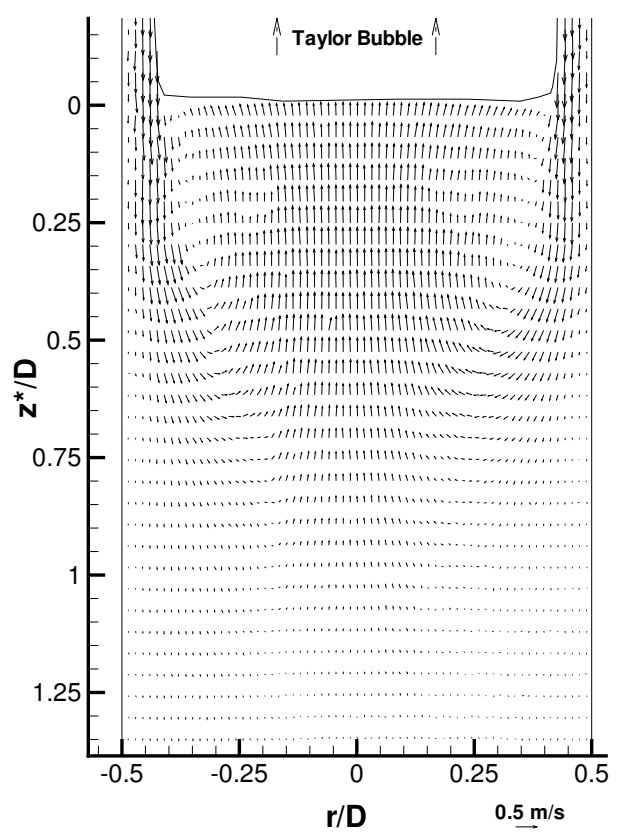

Figure 10. Instantaneous velocity field in the wake of a Taylor bubble rising in a $0.4 \mathrm{wt} \%$ solution

so the three dimensional flow field in the wake can be easily extrapolated by rotation of this plane. For the $0.4 \mathrm{wt} \%$, the liquid coming from the falling film smoothly expands after the bubble trailing edge inducing the formation of a closed donut shaped vortex in the wake of the bubble, that climbs with it.

As the Re decreases, the size of the wake also decreases. Due 
to the higher viscosity, the shear stresses exerted in the liquid are higher, which slows down faster the fluid coming from the liquid film, so it expands closer to the trailing edge.

If the slug velocity is subtracted to the velocity vectors, we obtain the flow field referred to a moving reference frame that rises with the same velocity of the bubble. In Figure 11 is represented an instantaneous flow field in the wake of a Taylor bubble rising in a $0.5 \mathrm{wt} \%$ solution, in a reference frame moving with the bubble velocity. From Figure 11 it becomes clear that the

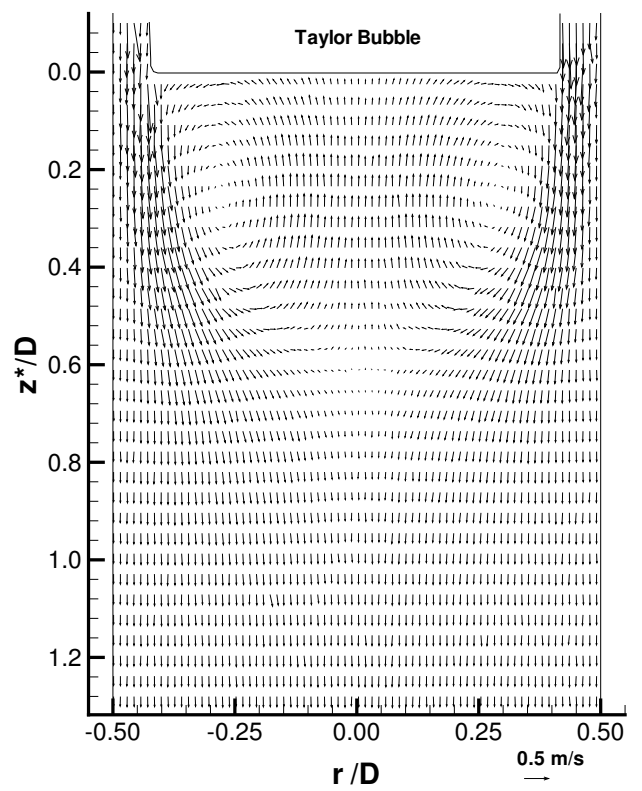

Figure 11. Instantaneous velocity field in the wake of a Taylor bubble rising in a $0.5 \mathrm{wt} \%$ solution in a reference frame moving with the bubble velocity

fluid coming from the liquid film expands around a recirculating zone that rises attached to the bubble.

The flow field in the wake of a Taylor bubble rising in a $0.6 \mathrm{wt} \%$ solution is represented in Figure 12. In the $0.6 \mathrm{wt} \%$ solution, the recirculation zone in the wake is not so clear. It is possible to see that the fluid coming from the liquid film expands much closer to the trailing edge than in the less viscous solutions and it goes up occupying the space that the bubble was occupying the instant before. The liquid that goes up in the center of the wake has now a velocity closer to the bubble velocity, while in the previous cases, the liquid was moving much faster. Figure 13 represents the same flow field of the Figure 12 but in a reference frame moving with the bubble velocity. It is possible to see that the fluid that comes from the liquid film quickly expands occupying all the column section with a severe velocity reduction. There is not a clear recirculation zone immediately after the bubble trailing edge, due to the small velocity amplitudes in this zone. It is possible that there is still liquid being transported in the wake, but in much smaller quantities and rotating velocity than in the less viscous cases.

The big differences between the Newtonian and nonNewtonian fluids wake's flow pattern appear in the higher viscosity solutions. For the 0.8 and $1.0 \mathrm{wt} \%$ solutions, there is a drastic change in the bubble trailing edge shape (Figure 4 and also in the wake flow pattern, which are fully described by

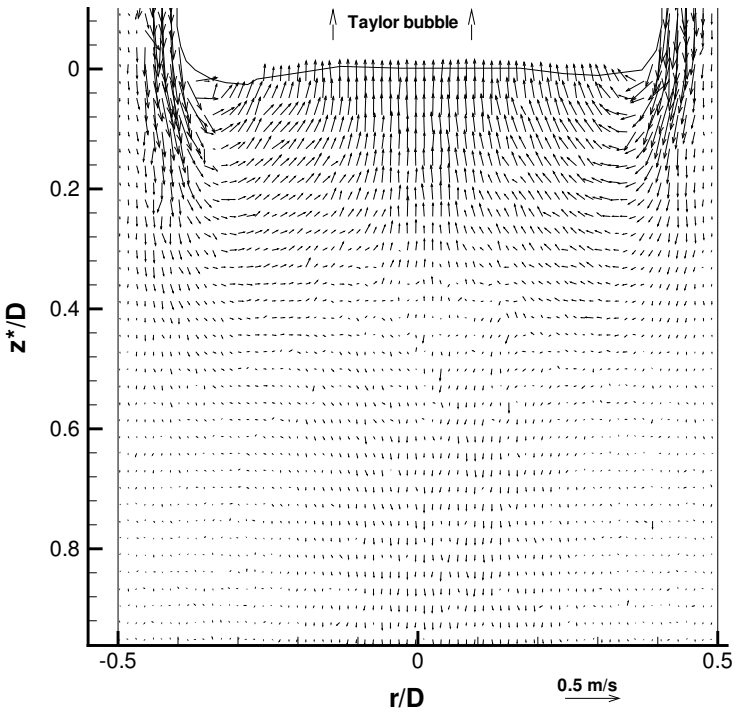

Figure 12. Instantaneous velocity field in the wake of a Taylor bubble rising in a $0.6 \mathrm{wt} \%$ solution in a fixed reference frame.

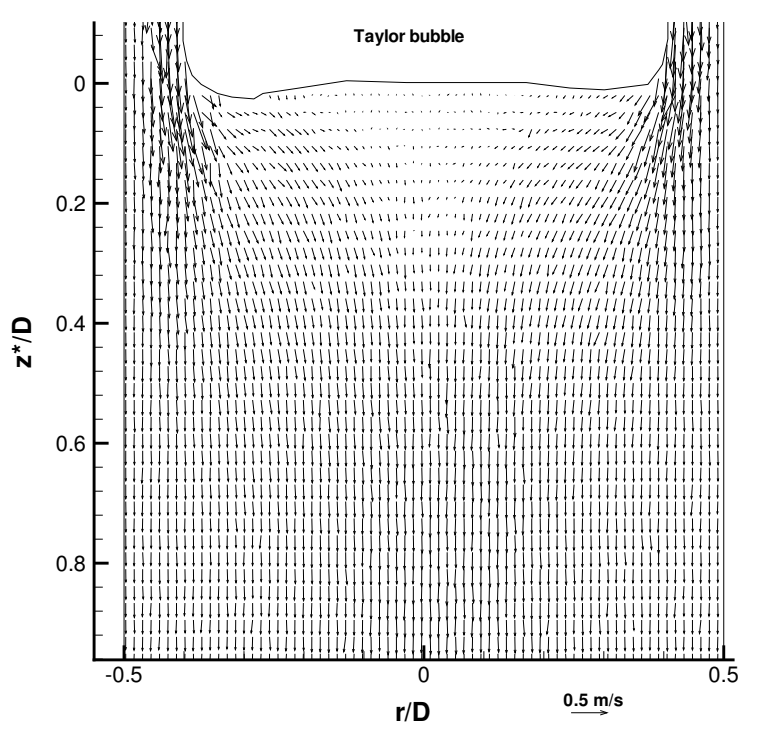

Figure 13. Instantaneous velocity field in the wake of a Taylor bubble rising in a $0.6 \mathrm{wt} \%$ solution in a reference frame moving with the bubble velocity.

Sousa et al [23]. In Figure 14 is represented the flow field in the trailing edge of a Taylor bubble rising in a $0.8 \mathrm{wt} \%$ solution. The flow around $0.8 \mathrm{wt} \%$ solution follows the tendency of the previous cases, where as the viscosity increases, the expansion of the fluid that comes from the liquid film happens closer to the trailing edge. The liquid immediately expands in the end of the liquid film, following the trailing edge shape and occupying the place left by the bubble. This radial movement of the liquid to the center of the column induces, by momentum diffusion, a rotational movement in the liquid that is below. The liquid rotates in the downward direction in the center of the column and upwards away from the center, creating what is called a negative wake, once the velocity in the center of the column is in the opposite direction of the bubble, contrasting with what happens in Newtonian fluids.

It becomes clear that in this case there is no liquid transported 


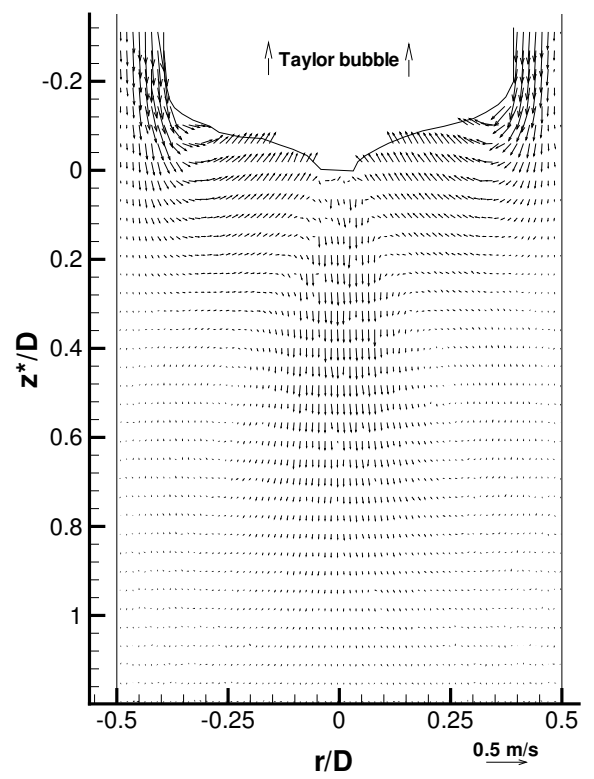

Figure 14. Instantaneous velocity field in the wake of a Taylor bubble rising in a $0.8 \mathrm{wt} \%$ solution in a fixed reference frame.

in the wake when we see the same flow field in a reference frame moving with the Taylor bubble (Figure 15). It is evident from

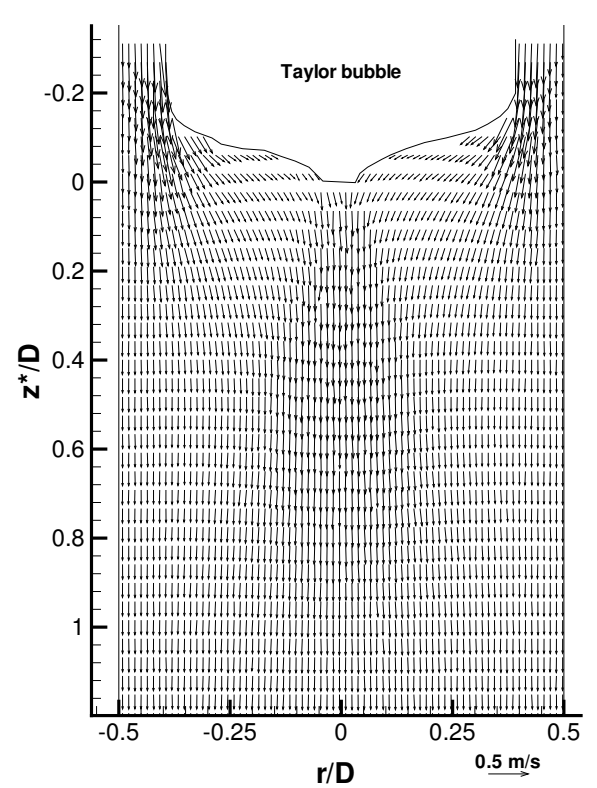

Figure 15. Instantaneous velocity field in the wake of a Taylor bubble rising in a $0.8 \mathrm{wt} \%$ solution in a reference frame moving with the bubble velocity.

Figure 15 that when seen in the moving reference frame, the liquid smoothly flows, expanding according to the Taylor bubble shape.

The flow around the trailing edge of a Taylor bubble rising in a $1.0 \mathrm{wt} \%$ solution is represented in Figure 16 and has the same pattern found for the $0.8 \mathrm{wt} \%$ solution but with a different shaped edge and with a lower downward velocity.

To compare the wake patterns and velocity magnitudes, the vertical component of the liquid velocity at $z *=0.2 D$ is repre-

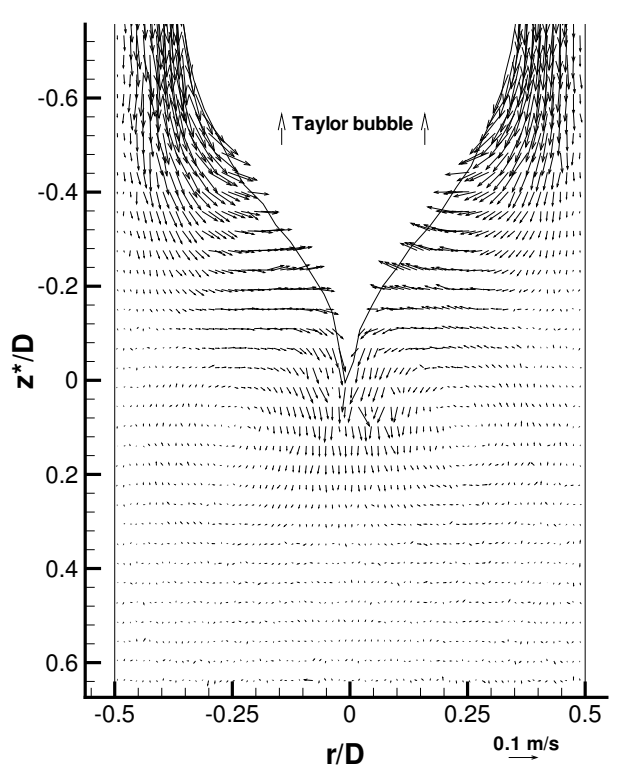

Figure 16. Instantaneous velocity field in the wake of a Taylor bubble rising in a $1.0 \mathrm{wt} \%$ solution in a fixed reference frame.

sented in Figure 17 for some of the cases studied. Figure 17 puts

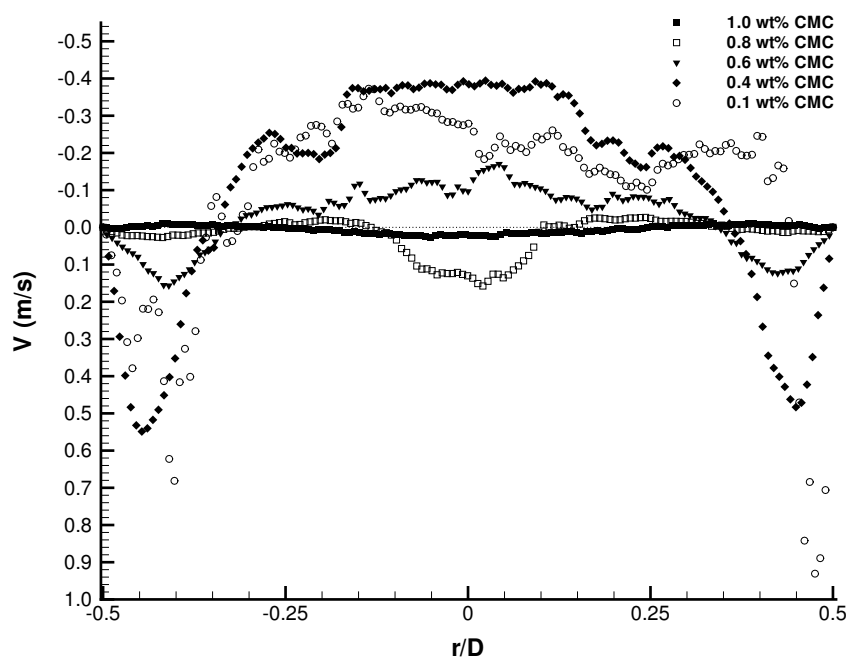

Figure 17. Vertical component of the liquid velocity in a fixed reference frame, along the line $z *=0.2 D$.

in evidence the occurrence of the negative wake for the 0.8 and $1.0 \mathrm{wt} \%$ solutions, due to the positive (downward) liquid velocity in the center of the column at $0.2 \mathrm{D}$ behind the trailing edge, which will have consequences in the coalescence phenomena.

\section{Conclusions}

Measurements of the flow field around Taylor bubbles rising in different solutions were made. This study shows the different wake flow patterns found in a $R e$ range between 4 and 254, which are essential to understand the coalescence mechanism between two slugs and the minimum distance between them above which there is no interaction between the Taylor bubbles. 
From these studies it becomes clear that for high values of $R e$ there should be interaction between two consecutive slugs even if they are separated by a large distance and that for the low values of $R e$, where the negative wake was found, it is not expected that the slugs coalesce.

\section{ACKNOWLEDGMENT}

The authors acknowledge the financial support given by F.C.T., SFRH/BD/3389/2000 and the Von Karman Institut for the facility. This work was also supported, via CEFT, by POCTI (FEDER).

\section{NOMENCLATURE}

$D$ Column diameter $[\mathrm{m}]$

De Deborah number $(\lambda / \tau)$ [dimensionless]

$L_{b} \quad$ Bubble length [m]

$n$ Carreau simplified model parameter

$r$ Radial position [m]

Re Reynolds number $\left(\rho U_{b} D / \mu_{b}\right)$ [dimensionless]

$T$ Temperature $\left.\left[{ }^{\circ} \mathrm{C}\right]\right)$

$U_{b} \quad$ Bubble velocity $[\mathrm{m} / \mathrm{s}]$

$V$ Vertical component of liquid velocity $[\mathrm{m} / \mathrm{s}]$

$z \quad$ Vertical distance to bubble nose [m]

$z * \quad$ Vertical distance to trailing edge center [m]

$\dot{\gamma}$ Shear rate $[1 / \mathrm{s}]$

$\dot{\gamma}_{b} \quad$ Bubble Shear rate $\left(U_{b} / D\right)[1 / \mathrm{s}]$

$\lambda$ Fluid characteristic time [s]

$\mu$ Liquid viscosity [Pa s]

$\mu_{0} \quad$ Liquid viscosity at zero shear rate [Pa s]

$\mu_{b} \quad$ Liquid viscosity at bubble shear rate [Pa s]

$\tau$ Flow characteristic time $\left(L_{b} / U_{b}\right)$ [s]

\section{REFERENCES}

[1] R. Moissis and P. Griffith. Entrance effects in a two-phase slug flow. J. Heat Transfer, Trans. ASME, Series C, 2:2939, 1962.

[2] Y. Taitel, D. Barnea, and A. Dukler. Modelling flow pattern transitions for steady upward gas-liquid flow in vertical tubes. AIChE Journal, 3:345-354, 1980.

[3] J. B. L. M. Campos and J. R. F. Guedes de Carvalho. An experimental study of the wake of gas slugs rising in liquids. J. Fluid Mechanics, 196:27-37, 1988.

[4] A. M. F. R. Pinto and J. B. L. M. Campos. Coalescence of two gas slugs rising in a vertical column of liquid. Chemical Engineering Science, 51:45-54, 1996.

[5] S. Polonsky, D. Barnea, and L. Shemer. Image processing procedure for analysing the motion of an elongated bubble rising in a vertical pipe. 8th International Symposium on Flow Visualisation, pages 117.1 - 117.10, 1998.

[6] S. Nogueira, I. Dias, A. M. F. R. Pinto, and M. L. Riethmuller. Liquid PIV measurements around a single gas slug rising through stagnant liquid in vertical pipes. In Bound Volume of Selected Papers of the "10th Symposium on Laser Techniques Applied to Fluid Dynamics". Springer, 2000.

[7] R. Van Hout, A. Gulitski, D. Barnea, and L. Shemer. Experimental investigation of the velocity field induced by a taylor bubble rising in stagnant water. International Jour- nal of Multiphase Flow, 28:579-596, 2001.

[8] J.D. Bugg and G. A. Saad. The velocity field around a taylor bubble rising in a stagnant viscous fluid: Numerical and experimental results. International Journal of Multiphase Flow, 28:791-803, 2002.

[9] G. Astarita and G. Apuzzo. Motion of gas bubbles in nonnewtonian liquids. AIChE Journal, 11:815-820, 1965.

[10] L. G. Leal, J. Skoog, and A. Acrivos. On the motion of gas bubbles in a viscoelastic liquid. The Canadian Journal of Chemical Engineering, 49:569-575, 1971.

[11] A. Acharya, R. A. Mashelkar, and J. Ulbrecht. Mechanics of bubble motion and deformation in non-newtonian media. Chemical Engineering Science, 32:863-872, 1977.

[12] O. Hassager. Negative wake behind bubbles in nonnewtonian liquids. Nature, 279:402-403, 1979.

[13] A. Acharya and J. J. Ulbrecht. Note on the influence of viscoelasticity on the coalescence rate of bubbles and drops. AIChE Journal, 24:348-351, 1978.

[14] D. De Kee, R. P. Chhabra, and A. Dajan. Motion and coalescence of gas bubbles in non-newtonian polymer solutions. Journal of Non-Newtonian Fluid Mechanics, 37:118, 1990.

[15] H. Z. Li, X. Frank, D. Funfschilling, and Youssef Mouline. Towards the understanding of bubble interactions and coalescence in non-newtonian fluids: A cognitive approach. Chemical Engineering Science, 56:6419-6425, 2001.

[16] D. Funfschilling and H. Z. Li. Flow of non-newtonian fluids around bubbles: PIV measurements and birefringe visualisation. Chemical Engineering Science, 56:11371141, 2001.

[17] P. S. Carew, N. H. Thomas, and A. B. Johnson. A physical based correlation for the effects of power law rheology and inclination on slug bubble rise velocity. Int. J. of Multiphase Flow, 21(6):1091-1106, 1995.

[18] L. Otten and A. S. Fayed. Pressure drop and drag reduction in two-phase non-newtonian slug flow. The Canadian Journal of Chemical Engineering, 54:111-114, 1976.

[19] R. G. Rosehart, E. Rhodes, and D. S. Scott. Studies of gas-liquid (non-newtonian) slug flow: Void fraction meter, void fraction and slug characteristics. The Chemical Engineering Journal, 10:57-64, 1975.

[20] Koichi Terasaka and Hideki Tsuge. Gas holdup for slug bubble flow of viscous liquids having a yield stress in bubble columns. Chemical Engineering Science, 58:513-517, 2003.

[21] R. Lindken and W. Merzkirch. A novel PIV technique for measurements in multi-phase flows and its application to two-phase bubbly flows. In 4th International Symposium on Particle Image Velocimetry, 2001.

[22] S. Nogueira, R. G. Sousa, A. M. F. R. Pinto, M. L. Riethmuller, and J. B. L. M. Campos. Simultaneous PIV and shadowgraphy in slug flow: A solution for optical problems. Experiments in Fluids, 35:598-609, 2003.

[23] R. G. Sousa, S. Nogueira, A. M. F. R. Pinto, M. L. Riethmuller, and J. B. L. M. Campos. Flow in the negative wake of a taylor bubble rising in a high concentrated shear thinning fluid: PIV measurements. Submitted, 2004.

[24] F. Scarano and M. L. Riethmuller. Iterative multigrid approach in PIV image processing with discrete window offset. Experiments in Fluids, 26:513-523, 1999. 